\title{
Clinical Case Study: Achieving Long-term Control of Insulin Resistance
}

\author{
Susan A. Cornell, BS, PharmD, CDE, CDM
}

\begin{abstract}
BACKGROUND: A total of 20.8 million people in the United States have diabetes, including 10.3 million adults over the age of 60 years, and more than 6 million people remain undiagnosed. Although diabetes is widely recognized as a prevalent and serious disease in managed care, current care is suboptimal, with less than $2 \%$ of American adults with diabetes receiving optimal quality of care.
\end{abstract}

OBJECTIVE: To review the various treatment interventions available in diabetes care, including the use of pay for performance (P4P) initiatives.

SUMMARY: In an effort to improve the current state of diabetes care, the NCQA's Health Plan Employer Data and Information Set (HEDIS) diabetes measure was developed as a means to better promote monitoring of various clinical markers in patients with this disease. This measure has been employed in P4P initiatives across the country by granting incentives to providers who have a prespecified proportion of their patients with diabetes meeting the measure. Likewise, to improve outcomes, many experimental and recently approved treatment options for diabetes target different processes in the course of the disease.

CONCLUSIONS: An effective program for the management of a diabetes patient population must be multidisciplinary, coordinating the efforts of many different levels of health care providers. Furthermore, components commonly incorporated in P4P initiatives, such as patient self-management education, provider contact, and the use of the American Diabetes Association standards of care measures for screening and lab levels, are imperative to the success of a diabetes management program.

KEYWORDS: P4P, Diabetes, NCQA, HEDIS, A1C, Glycemic control, Postprandial, Lifestyle modification, Patient education

J Manag Care Pharm. 2007;13(2)(suppl S-b)S11-S15
Author

SUSAN A. CORNELL, BS, PharmD, CDE, CDM, is an assistant professor Department of Pharmacy Practice, Midwestern University Chicago College of Pharmacy, Downers Grove, Illinois.

AUTHOR CORRESPONDENCE: Susan A. Cornell, BS, PharmD, CDE, CDM, Assistant Professor, Department of Pharmacy Practice, Midwestern University Chicago College of Pharmacy, 555 W. 31st St., Downers Grove, IL 60515. Tel: (630) 515-6191; Fax: (630) 575-6103;

E-mail:scorne@midwestern.edu

Copyright $\odot$ 2007, Academy of Managed Care Pharmacy. All rights reserved. $\square$ iabetes, a growing threat to our nation's health, is currently diagnosed in an estimated 20.8 million Americans, and approximately 35 million Americans have prediabetes. ${ }^{1}$ In addition, this multiorgan disease is a growing health problem for future generations, with a reported $7 \%$ to $45 \%$ increase in children with type 2 diabetes. ${ }^{2}$

In an effort to control this epidemic of diabetes, pay for performance (P4P) initiatives, such as those based on the National Committee for Quality Assurance's (NCQA) Health Plan Employer Data and Information Set (HEDIS) diabetes care measure, have emerged. New diabetes treatment strategies, including these P4P initiatives, have placed an increasing emphasis on tight glycemic control. Still, despite these efforts, fewer than 2\% of American adults with diabetes receive optimal quality of care, and national averages of blood glucose levels are well above desired target goals. ${ }^{3,4}$

Diabetes is a complex condition, often accompanied by significant comorbidities. This complexity, coupled with the tendency for patients to be diagnosed late in course of illness, are just 2 possible reasons for this alarming trend and the current state of diabetes management. An examination of a case study of a 62-year-old male with type 2 diabetes and multiple comorbidities will be used to illustrate the impact of these factors on diabetes management.

The case study highlights the role of pharmacological agents with different mechanisms of action, in addition to nutrition and lifestyle changes, and emphasizes the importance of patient education and empowerment in the treatment of diabetes. All of these factors can contribute to improvements in P4P scores.

A focus on postprandial hyperglycemia, in addition to fasting blood glucose excursions, may serve to improve the current state of diabetes care. Adding such recently introduced agents as the incretin hormones, glucagon-like protein-1 (GLP-1) analogs, and dipeptidyl peptidase-IV (DPP-IV) inhibitors to the existing treatment plans may prove more beneficial in improving glycemic control than traditional agents. These therapies target different processes in the course of diabetes, exerting their effects on postprandial glucose excursions. The use of individually tailored therapy, employing the most effective pharmacologic agents possible in conjunction with nutrition and lifestyle changes, remains the best option. Best practices such as these can be enhanced through $\mathrm{P} 4 \mathrm{P}$ initiatives to further the effectiveness of diabetes therapy in managed care.

\section{Incidence and Prevalence of Diabetes}

Diabetes is one of the most prevalent diseases in the United States, with one person in America being diagnosed every 25 seconds. ${ }^{1}$ A total of 20.8 million people in the United States have diabetes, including 10.3 million adults over the age of 60 years, and more 


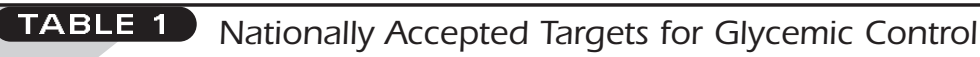

\begin{tabular}{l|c|c|c}
\hline & AlC (\%) & FPG (mg/dL & PPG (mg/dL) \\
\hline ADA & $<7.0$ & $90-130$ & $<1801-2$ hours \\
\hline AACE & $<6.5$ & $<110$ & $<1402$ hours \\
\hline Normal & $<6.0$ & $<110$ & $<140$ \\
\hline
\end{tabular}

Diabetes Care. 2006;29(suppl 1):S4-S39.

$A 1 C=$ glycosylated hemoglobin; $A A C E=$ American Association of Clinical Endocrinologists; $A D A=$ American Diabetes Association; FPG=fasting plasma glucose; $P P G=$ postprandial blood glucose.

than 6 million people remain undiagnosed. ${ }^{1}$ Mortality rates from diabetes and its related conditions, such as cardiovascular disease, highlight the seriousness of the this disease and contribute to it being the sixth leading cause of death listed on U.S. death certificates in 2002. ${ }^{6}$ Furthermore, the problem of diabetes is growing, as approximately 35 million people in the United States have prediabetes. ${ }^{1}$ This epidemic is not confined to adults or the elderly-reports indicate a $7 \%$ to $45 \%$ increase in children with type 2 diabetes. ${ }^{2}$

\section{Current Trends in Diabetes Management}

Although diabetes is widely recognized as a prevalent and serious disease in managed care, current care is suboptimal. In fact, fewer than $2 \%$ of American adults with diabetes are receiving optimal quality of care. ${ }^{3}$

Tight glycemic control, a fasting blood glucose goal of $<110$ $\mathrm{mg} / \mathrm{dL}$ and a 2-hour postprandial goal of $<140 \mathrm{mg} / \mathrm{dL}$, (Table 1) ${ }^{5}$ is a key component of diabetes management, since the maintenance of blood glucose within a narrow target range can minimize or prevent the microvascular and macrovascular complications associated with the disease. While this concept is universally accepted, the national averages of blood glucose levels remain well above desired target goals, indicating the need for improved management and care. Recent estimates of the U.S. averages were $>200 \mathrm{mg} / \mathrm{dL}$ for fasting plasma glucose (FPG), $>300$ $\mathrm{mg} / \mathrm{dL}$ for postprandial blood glucose (PPG), and greater than 9.5\% for glycosylated hemoglobin (A1C). ${ }^{4}$

In an effort to improve the current state of diabetes care, the NCQA's HEDIS diabetes measure was developed as a means to better promote monitoring of various clinical markers in patients with this disease. The HEDIS diabetes measure evaluates the percentage of plan members with diabetes (aged 18-75 years) with AlC screening; AlC controlled to $<9.0 \%$ (which will change to $<7.0 \%$ in 2007); low-density lipoprotein cholesterol (LDL-C) screening; LDL-C controlled to $<100 \mathrm{mg} / \mathrm{dL}$; eye examination; and microalbuminuria screening. ${ }^{8}$ This measure has been employed in P4P initiatives across the country by granting incentives to providers who have a prespecified proportion of their patients with diabetes meeting the measure.

Over the past decade, providers in the United States have

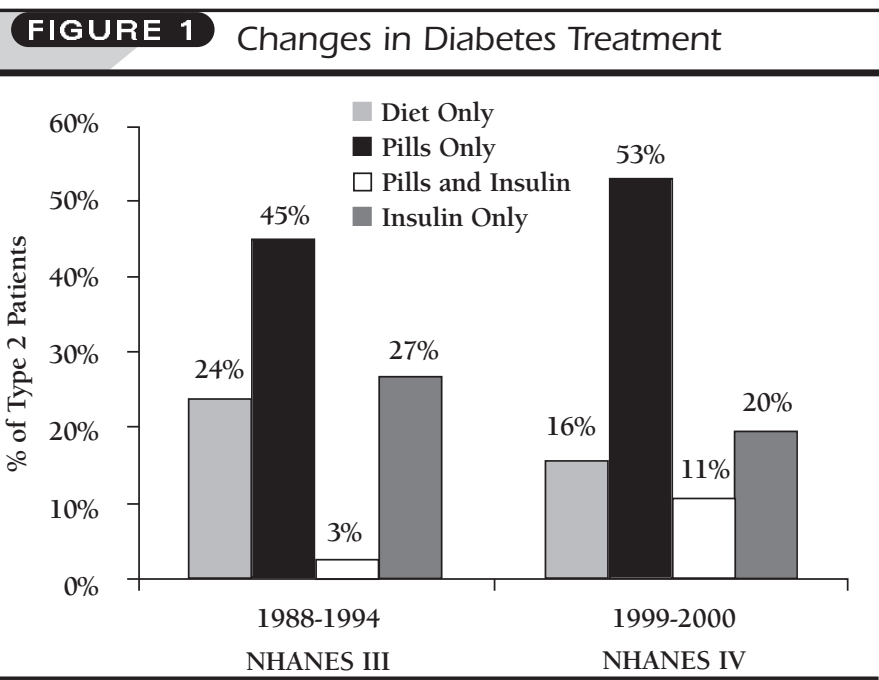

Koro CE et al. Diabetes Care. 2004;27:17-19.

NHANES = National Health and Nutrition Examination Survey.

shifted the treatment paradigm toward a strategy that incorporates the use of more pharmacologic agents and fewer dietary or lifestyle interventions or insulin therapy alone. The use of pharmacotherapy alone in treatment of diabetes increased from $45 \%$ to $53 \%$ in type 2 patients, while the use of dietary interventions and insulin alone decreased from 24\% and 27\% to 16\% and 20\%, respectively, in patients with type 2 diabetes (Figure 1). ${ }^{9}$

These changes in diabetes pharmacotherapy do not appear to have resulted in improved levels of glycemic control. Indeed, the proportion of patients achieving the American Diabetes Association's (ADA's) recommended AlC level of $<7.0 \%$ as measured in the National Health and Nutrition Examination Survey (NHANES) III and IV actually decreased from 44.5\% to $35.8 \%$ between the years 1994 and 2000, indicating the need to continue to improve education and self-management efforts. ${ }^{9}$ It is possible that increasing reliance solely on pharmacological measures, especially monotherapy, resulted in an overall worsening of the condition in patients, as demonstrated by the trend toward less-effective glucose control. 


\section{Clinical Case Study}

Examination of a case study of a 62-year-old male with type 2 diabetes for 4 years helps to demonstrate the challenges inherent in the treatment of patients struggling with the disease. It is important to remember that the ADA estimates that by the time a patient with type 2 diabetes is finally diagnosed, they have actually had the disease for approximately 9 years. ${ }^{10}$ Therefore, from the time of diagnosis, treatment plans need to be aggressive in order to optimally manage this disease, since most patients already have complications resulting from this delay in diagnosis.

The patient's laboratory and physical findings in this study are outlined in Table 2. As with most diabetes patients, this particular patient has concurrent cardiovascular disease, specifically hypertension and dyslipidemia. To assist in the management of these conditions, the patient had been prescribed glyburide $5 \mathrm{mg}$ two times daily, metformin $850 \mathrm{mg}$ three times daily, an ACE inhibitor, a diuretic, and a low-dose statin.

Illustrating the complexity of treating such a high-maintenance disease, the patient acknowledged having a self-monitoring blood glucose (SMBG) meter but reported having "no time" to check his blood glucose levels. In addition, the patient admitted to a sedentary lifestyle, which led to his gradual increase in weight since diagnosis. He also described poor nutritional habits, such as eating fast food more than 3 times a week, thereby adding to poor glucose control and a need for a more aggressive treatment plan.

Diabetes self-management education (DSME) is the cornerstone of optimal management of this disease. As such, patient selfmanagement education and quality physician contact commonly incorporated in $\mathrm{P} 4 \mathrm{P}$ initiatives may benefit this patient. Motivational and behavioral change strategies that support lifestyle improvements would likely lead to better glucose control, enhanced quality of life, and an increased likelihood of treatment success. In addition, at the time of diagnosis, the patient did not receive a dilated eye exam, a monofilament foot exam, or screening for kidney disease, as mandated in the ADA standards of care. This demonstrates the need and value of P4P and other initiatives that can increase the likelihood of better managing patients with diabetes.

To improve the patient's overall health, treatment goals include reducing his $\mathrm{AlC}$ to $<7 \%$ (preferably $<6.5 \%$ ), and reducing LDL-C to $<100 \mathrm{mg} / \mathrm{dL}$. In addition, the patient is scheduled for regular dilated eye exams, monofilament foot exams, and screening for kidney disease to ensure consistency in monitoring and care. A self-management diabetes education program was also implemented for this patient to better manage his diabetes by increasing his SMBG meter use to twice daily, improving his nutritional habits, and increasing his physical activity. These common P4P-related activities ultimately served as the base for the patient's diabetes management.

Although lifestyle played an important role in the progression of this patient's disease, his diabetes remained uncontrolled despite pharmacological treatment with glyburide and metformin.

\section{TABLE 2 Physical and Laboratory Findings for a 62-Year-Old Male}

\begin{tabular}{l|l}
\hline Physical Findings & \\
\hline Weight & $227 \mathrm{lbs}$ \\
\hline BMI & 30.8 \\
\hline Blood pressure & $122 / 78 \mathrm{~mm} \mathrm{Hg}$ \\
\hline Laboratory Findings & \\
\hline AlC & $9.1 \%$ \\
\hline FPG & $168 \mathrm{mg} / \mathrm{dL}$ \\
\hline TC & $211 \mathrm{mg} / \mathrm{dL}$ \\
\hline LDL-C & $138 \mathrm{mg} / \mathrm{dL}$ \\
\hline HDL-C & $31 \mathrm{mg} / \mathrm{dL}$ \\
\hline TG & $210 \mathrm{mg} / \mathrm{dL}$ \\
\hline
\end{tabular}

$A 1 C=$ glycosylated hemoglobin; $B M I=$ body mass index; $F P G=$ fasting plasma glucose; HDL-C = high-density lipoprotein cholesterol; $L D L-C=l o w$-density lipoprotein cholesterol; TC=total cholesterol; $T G=$ triglyceride.

Until the past decade, few pharmacologic options existed that focused on treating the cause of diabetes as opposed to the symptoms. Additionally, diabetes is a multifaceted disease requiring different agents that work on different organs. Recent pharmacological advancements have introduced new agents that may play an important role in improving the glucose control of this patient as well as that of others. In order to determine whether a switch in therapy is necessary, the current regimen must be evaluated to determine the drugs' efficacy in reducing the patient's AlC to $<7 \%$. If the current pharmacologic agents (glyburide, metformin) at their current dose are determined to have suboptimal effectiveness, action must be taken either in considering dose adjustment or adding or switching agents to reduce and maintain AlC levels. In terms of switching agents, alternative conventional therapies for this particular patient include alpha glucosidase inhibitors, glitinides, or thiazolidinediones (TZDs), while emerging therapies include GLP-1 analogs or DPP-IV inhibitors.

\section{Emerging Therapies in Diabetes Management}

Many experimental and recently approved treatment options for diabetes target different processes in the course of the disease. In particular, a focus on improvement in the regulation of the gastrointestinal motility and the postprandial hyperglycemia that accompany the disease may serve to improve the current state of diabetes care. Postprandial hyperglycemia is an important predictor of cardiovascular disease and needs to be accurately monitored to properly adjust dietary intake, oral medications, and insulin..$^{11,12}$ Postprandial blood glucose levels are required to accurately adjust bolus insulin doses. ${ }^{13}$ In addition, the postprandial glucose values tend to correlate better with AlC than fasting glucose, especially at values near the $7 \%$ range. ${ }^{14} \mathrm{~A}$ recent study by Monnier et al. 
suggests that approximately $70 \%$ of the glucose that contributes to an $\mathrm{AlC}$ of $7 \%$ comes from the after-meal or postprandial glucose level. ${ }^{15}$

Emerging therapies in diabetes care include the GLP-1 analogs and DPP-IV inhibitors, which each exert their effect on different portions of the same pathway in the mechanism of the disease. The GLP-1 analogs, or incretin mimetics, act by mimicking the action of GLP-1, an incretin hormone secreted in the L-cells of the intestinal mucosa that is released in response to food and binds to the pancreatic $\beta$-cell to regulate insulin secretion, slow gastric emptying, and inhibit gluconeogenesis. Agents from this category include pramlintide and exenatide, which are approved for use and are administered as a subcutaneous injection prior to major meals, and liraglutide, which is in development and not available for use. ${ }^{16}$

While GLP- 1 analogs affect the processes involved in diabetes by mimicking the action of GLP-1 and enhancing natural GLP-1derived processes, DPP-IV inhibitors act by preventing the action of DPP-IV, the key enzyme involved in the degradation of GLP-1. ${ }^{17}$ This results in an increase in (1) an endogenous GLP-1 in response to a meal, (2) suppression of glucagon release, and (3) improvement in peripheral glucose utilization. The DPP-IV inhibitors enhance the long-term effects of GLP-1, such as increasing insulin synthesis and promoting the preservation, neogenesis, and restoration of $\beta$-cells (Figure 2). DPP-IV inhibitors are oral agents and include sitagliptin, which was recently approved for use in the United States, and vildagliptin and saxagliptin, which are in development. ${ }^{18}$

\section{Conclusions: Best Practices in Diabetes Management}

An effective program for the management of a diabetes patient population must be multidisciplinary, coordinating the efforts of a primary care provider, endocrinologist, diabetes educator, dietitian, pharmacist, and support staff. Components commonly incorporated in $\mathrm{P} 4 \mathrm{P}$ initiatives, such as patient self-management education, provider contact, and the use of the ADA standards of care measures for screening and lab levels, are imperative to the success of the program. As an example, organizations such as Kaiser Permanente; Health Partners in Minneapolis, Minnesota; and the Family Medicine Center at the University of Oklahoma Health Science Center, Oklahoma City, have designed and implemented multidisciplinary diabetes management programs that have been met with success to date.

Diabetes management must focus on establishing and maintaining glycemic control, reducing the impact of comorbid conditions, and minimizing or preventing long-term complications of diabetes. Treatment should be multifaceted and include aggressive lifestyle modification as well as safe and efficacious pharmacologic agents. The HEDIS measure for comprehensive diabetes management serves as an excellent benchmark for ideal care. Whether part of a P4P program or not, achievement of the HEDIS measure for comprehensive diabetes management can favorably improve outcomes.

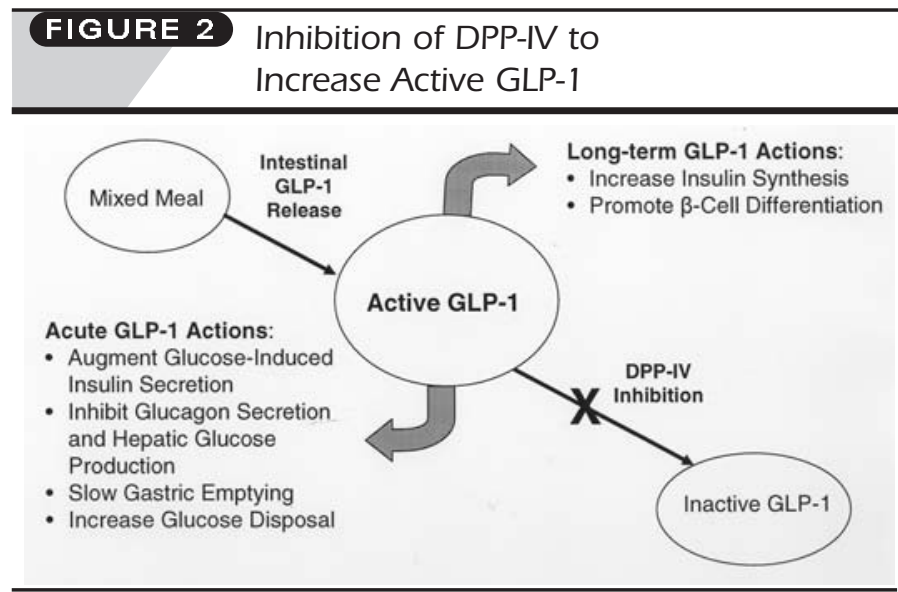

Drucker DJ. Diabetes Care. 2003;26:2929-40.

DPP-IV = dipeptidyl peptidase $-I V ; G L P-1=$ glucagon-like protein-1.

\section{DISCLOSURES}

This article is based on a presentation given by the author at a symposium, "Pay for Performance: Where's the Return?" held October 4, 2006, at the Academy of Managed Care Pharmacy's 2006 Educational Conference in Chicago, Illinois. The symposium was supported by an educational grant from Merck \& Co., Inc. The author discloses that she has received an honorarium from Merck \& Co., Inc. for participation in the symposium and this supplement. She discloses no potential bias or conflict of interest relating to this article.

\section{REFERENCES}

1. Centers for Disease Control and Prevention. National diabetes fact sheet: general information and national estimates on diabetes in the United States, 2005. Available at: http://www.cdc.gov/DIABETES/pubs/pdf/ndfs_2005.pdf. Accessed July 25, 2006

2. Mokdad AH, Ford ES, Bowman BA, et al. Diabetes trends in the U.S. 1990-1998. Diabetes Care. 2000;23(9):1278-83.

3. Beckles GL, Engelgau MM, Narayan KM, Herman WH, Aubert RE, Williamson DF. Population-based assessment of the level of care among adults with diabetes in the U.S. Diabetes Care. 1998;21(9):1432-38.

4. Saaddine JB, Engelgau MM, Beckles GL, et al. A diabetes report card for the United States: quality of care in the 1990s. Ann Intern Med. 2002;136(8):565-74.

5. Standards of medical care in diabetes-2006. Diabetes Care. 2006;29 (suppl 1):S4-S42.

6. Wyne KL. The need for reappraisal of type 2 diabetes mellitus management. Postgrad Med. May 2003;spec. no.:5-14.

7. Avignon A, Radauceanu A, Monnier L. Nonfasting plasma glucose is a better marker of diabetic control than fasting plasma glucose in type 2 diabetes. Diabetes Care. 1997;20(12):1822-26.

8. National Committee for Quality Assurance. State of Health Care Quality 2005. Available at: http://www.ncqa.org/Docs/SOHCQ_2005.pdf. Accessed November 19, 2006.

9. Koro CE, Bowlin SJ, Bourgeois N, Fedder DO. Glycemic control from 1988 to 2000 among U.S. adults diagnosed with type 2 diabetes: a preliminary report. Diabetes Care. 2004;27(1):17-20.

10. U.K. Prospective Diabetes Study 16. Overview of 6 years' therapy of type II diabetes: a progressive disease. U.K. Prospective Diabetes Study Group. Diabetes. 1995;44(11):1249-58. 
11. Avignon A, Radauceanu A, Monnier L. Nonfasting plasma glucose is a better marker of diabetic control than fasting plasma glucose in type 2 diabetes. Diabetes Care. 1997;20(12):1822-26.

12. Bonora E, Calcaterra F, Lombardi S, et al. Plasma glucose levels throughout the day and $\mathrm{HbA}(\mathrm{lc})$ interrelationships in type 2 diabetes: implications for treatment and monitoring of metabolic control. Diabetes Care. 2001;24(12): 2023-29.

13. Buse JB. Should postprandial glucose be routinely measured and treated to a particular target? No! Diabetes Care. 2003;26(5):1615-18.

14. Davidson J. Should postprandial glucose be measured and treated to a particular target? Yes. Diabetes Care. 2003;26(6):1919-21.
15. Monnier L, Lapinski H, Colette C. Contributions of fasting and postprandial plasma glucose increments to the overall diurnal hyperglycemia of type 2 diabetic patients. Diabetes Care. 2003:26;881-85.

16. Byetta [package insert]. San Diego, CA: Amylin Pharmaceuticals; 2006.

17. Drucker DJ. Enhancing incretion action for the treatment of type 2 diabetes. Diabetes Care. 2003;26:2929-40.

18. Januvia [package insert]. Whitehouse Station, NJ: Merck \& Co; 2006. 